\title{
OBSERVATION OF SUPERNOVA REMNANT IC 443 WITH THE FERMI LARGE AREA TELESCOPE
}

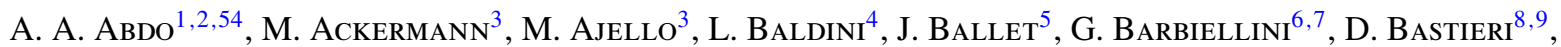

B. M. BAughman ${ }^{10}$, K. BeChtol ${ }^{3}$, R. Bellazzini ${ }^{4}$, B. Berenji ${ }^{3}$, R. D. BlandFord ${ }^{3}$, E. D. Bloom ${ }^{3}$, E. Bonamente ${ }^{11,12}$, A. W. Borgland ${ }^{3}$, J. Bregeon ${ }^{4}$, A. Brez ${ }^{4}$, M. Brigida ${ }^{13,14}$, P. Bruel ${ }^{15}$, T. H. BurnetT ${ }^{16}$, S. Buson ${ }^{9}$, G. A. Caliandro ${ }^{13,14}$, R. A. Cameron ${ }^{3}$, P. A. Caraveo ${ }^{17}$, J. M. Casandian ${ }^{5}$, C. CeCchi ${ }^{11,12}$, Ö. Çelik ${ }^{18,19,20}$, A. Chekhtman ${ }^{1,21}$, C. C. Cheung ${ }^{18}$, J. Chiang ${ }^{3}$, A. N. Cillis ${ }^{18}$, S. Ciprini ${ }^{11,12}$, R. Claus ${ }^{3}$, J. Cohen-TANugi ${ }^{22}$, L. R. Cominsky ${ }^{23}$, J. ConRad ${ }^{24,25,55}$, S. Cutini ${ }^{26}$, C. D. Dermer ${ }^{1}$, A. De Angelis ${ }^{27}$, F. De Palma ${ }^{13,14}$, E. do Couto e Silva ${ }^{3}$, P. S. Drell ${ }^{3}$, A. Drlica-Wagner ${ }^{3}$, R. Dubois ${ }^{3}$, D. Dumora ${ }^{28,29}$, C. FArnier ${ }^{22}$, C. FAVuzzi ${ }^{13,14}$, S. J. Fegan ${ }^{15}$, W. B. Focke ${ }^{3}$, P. Fortin ${ }^{15}$, M. Frailis ${ }^{27}$, Y. FuKaZawa ${ }^{30}$, S. FunK ${ }^{3}$, P. Fusco ${ }^{13,14}$, F. Gargano ${ }^{14}$, D. Gasparrini ${ }^{26}$, N. Gehrels ${ }^{18,31}$, S. Germani ${ }^{11,12}$, G. Giavitto ${ }^{32}$, B. Giebels ${ }^{15}$, N. Giglietto ${ }^{13,14}$, F. Giordano ${ }^{13,14}$, T. Glanzman ${ }^{3}$, G. Godfrey ${ }^{3}$, I. A. Grenier ${ }^{5}$, M.-H. Grondin ${ }^{28,29}$, J. E. Grove ${ }^{1}$,

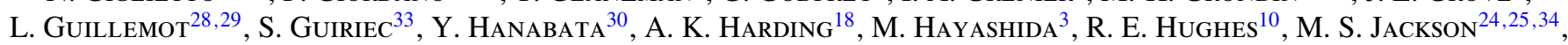

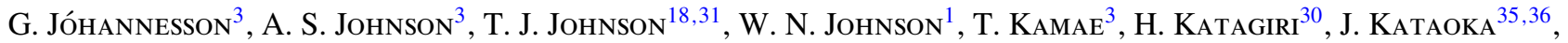

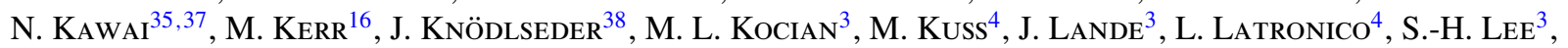

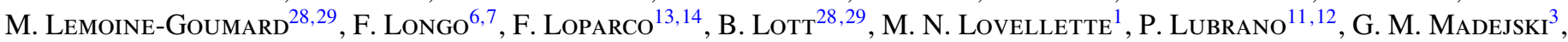
A. Makeev ${ }^{1,21}$, M. N. Mazziotta ${ }^{14}$, C. Meurer ${ }^{24,25}$, P. F. Michelson ${ }^{3}$, W. Mitthumsiri ${ }^{3}$, A. A. Moiseev ${ }^{19,31}$, C. Monte ${ }^{13,14}$, M. E. Monzani ${ }^{3}$, A. Morselli ${ }^{39}$, I. V. Moskalenko ${ }^{3}$, S. Murgia ${ }^{3}$, T. Nakamori ${ }^{35}$, P. L. Nolan ${ }^{3}$, J. P. Norris ${ }^{40}$, E. Nuss ${ }^{22}$,

T. Ohsugi ${ }^{30}$, E. Orlando ${ }^{41}$, J. F. Ormes ${ }^{40}$, M. Ozaki ${ }^{42}$, D. Paneque ${ }^{3}$, J. H. Panetta ${ }^{3}$, D. Parent 28,29 , V. Pelassa ${ }^{22}$, M. Pepe ${ }^{11,12}$, M. Pesce-Rollins ${ }^{4}$, F. Piron ${ }^{22}$, T. A. Porter ${ }^{43}$, S. Rainò ${ }^{13,14}$, R. Rando ${ }^{8,9}$, M. Razzano ${ }^{4}$, A. Reimer ${ }^{3,44}$,

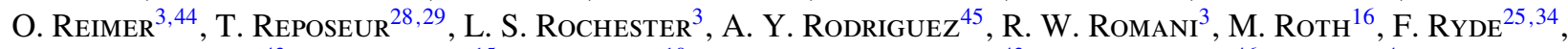

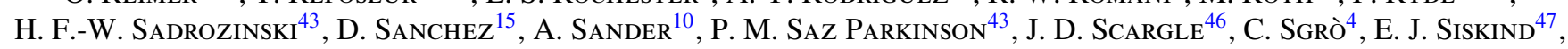
D. A. Smith $^{28,29}$, P. D. Smith ${ }^{10}$, G. Spandre ${ }^{4}$, P. Spinelli ${ }^{13,14}$, M. S. Strickman ${ }^{1}$, A. W. Strong ${ }^{41}$, D. J. Suson ${ }^{48}$, H. Tajima $^{3}$,

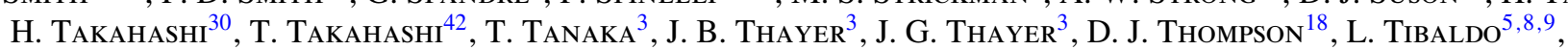

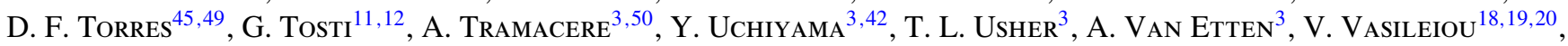

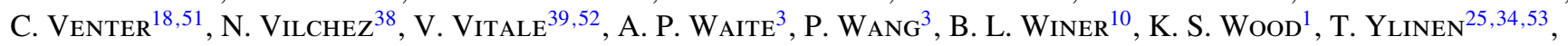
AND M. ZIEGLER ${ }^{43}$

${ }^{1}$ Space Science Division, Naval Research Laboratory, Washington, DC 20375, USA

${ }^{2}$ National Academy of Sciences, Washington, DC 20001, USA

${ }^{3}$ W. W. Hansen Experimental Physics Laboratory, Kavli Institute for Particle Astrophysics and Cosmology, Department of Physics and SLAC National Accelerator Laboratory, Stanford University, Stanford, CA 94305, USA; kamae@ slac.stanford.edu; shia520@ stanford.edu

${ }^{4}$ Istituto Nazionale di Fisica Nucleare, Sezione di Pisa, I-56127 Pisa, Italy

${ }^{5}$ Laboratoire AIM, CEA-IRFU/CNRS/Université Paris Diderot, Service d'Astrophysique, CEA Saclay, 91191 Gif sur Yvette, France

${ }^{6}$ Istituto Nazionale di Fisica Nucleare, Sezione di Trieste, I-34127 Trieste, Italy

${ }^{7}$ Dipartimento di Fisica, Università di Trieste, I-34127 Trieste, Italy

${ }^{8}$ Istituto Nazionale di Fisica Nucleare, Sezione di Padova, I-35131 Padova, Italy

${ }^{9}$ Dipartimento di Fisica "G. Galilei," Università di Padova, I-35131 Padova, Italy

${ }^{10}$ Department of Physics, Center for Cosmology and Astro-Particle Physics, The Ohio State University, Columbus, OH 43210, USA

${ }^{11}$ Istituto Nazionale di Fisica Nucleare, Sezione di Perugia, I-06123 Perugia, Italy

${ }^{12}$ Dipartimento di Fisica, Università degli Studi di Perugia, I-06123 Perugia, Italy

${ }^{13}$ Dipartimento di Fisica “M. Merlin” dell’Università e del Politecnico di Bari, I-70126 Bari, Italy; francesco.giordano@ba.infn.it

${ }^{14}$ Istituto Nazionale di Fisica Nucleare, Sezione di Bari, 70126 Bari, Italy

${ }^{15}$ Laboratoire Leprince-Ringuet, École polytechnique, CNRS/IN2P3, Palaiseau, France

${ }^{16}$ Department of Physics, University of Washington, Seattle, WA 98195-1560, USA

${ }^{17}$ INAF-Istituto di Astrofisica Spaziale e Fisica Cosmica, I-20133 Milano, Italy

${ }_{18}$ NASA Goddard Space Flight Center, Greenbelt, MD 20771, USA

${ }^{19}$ Center for Research and Exploration in Space Science and Technology (CRESST), NASA Goddard Space Flight Center, Greenbelt, MD 20771, USA

${ }^{20}$ Department of Physics and Center for Space Science and Technology, University of Maryland, Baltimore County, Baltimore, MD 21250, USA

${ }^{21}$ Center for Earth Observing and Space Research, George Mason University, Fairfax, VA 22030, USA

${ }^{22}$ Laboratoire de Physique Théorique et Astroparticules, Université Montpellier 2, CNRS/IN2P3, Montpellier, France

${ }^{23}$ Department of Physics and Astronomy, Sonoma State University, Rohnert Park, CA 94928-3609, USA

${ }^{24}$ Department of Physics, Stockholm University, AlbaNova, SE-106 91 Stockholm, Sweden

25 The Oskar Klein Centre for Cosmoparticle Physics, AlbaNova, SE-106 91 Stockholm, Sweden

${ }^{26}$ Agenzia Spaziale Italiana (ASI) Science Data Center, I-00044 Frascati (Roma), Italy

27 Dipartimento di Fisica, Università di Udine and Istituto Nazionale di Fisica Nucleare, Sezione di Trieste, Gruppo Collegato di Udine, I-33100 Udine, Italy

${ }^{28}$ Université de Bordeaux, Centre d'Études Nucléaires Bordeaux Gradignan, UMR 5797, Gradignan, 33175, France

${ }^{29}$ CNRS/IN2P3, Centre d'Études Nucléaires Bordeaux Gradignan, UMR 5797, Gradignan, 33175, France

${ }^{30}$ Department of Physical Sciences, Hiroshima University, Higashi-Hiroshima, Hiroshima 739-8526, Japan

${ }^{31}$ Department of Physics and Department of Astronomy, University of Maryland, College Park, MD 20742, USA

${ }^{32}$ Istituto Nazionale di Fisica Nucleare, Sezione di Trieste, and Università di Trieste, I-34127 Trieste, Italy

${ }^{33}$ Center for Space Plasma and Aeronomic Research, University of Alabama in Huntsville, Huntsville, AL 35899, USA

${ }^{34}$ Department of Physics, Royal Institute of Technology (KTH), AlbaNova, SE-106 91 Stockholm, Sweden

${ }^{35}$ Department of Physics, Tokyo Institute of Technology, Meguro City, Tokyo 152-8551, Japan

${ }^{36}$ Research Institute for Science and Engineering, Waseda University, 1-104 Totsukamachi, Shinjuku-ku, Tokyo, 169-8050, Japan

${ }^{37}$ Cosmic Radiation Laboratory, Institute of Physical and Chemical Research (RIKEN), Wako, Saitama 351-0198, Japan

${ }^{38}$ Centre d'Étude Spatiale des Rayonnements, CNRS/UPS, BP 44346, F-30128 Toulouse Cedex 4, France

${ }^{39}$ Istituto Nazionale di Fisica Nucleare, Sezione di Roma “Tor Vergata," I-00133 Roma, Italy 


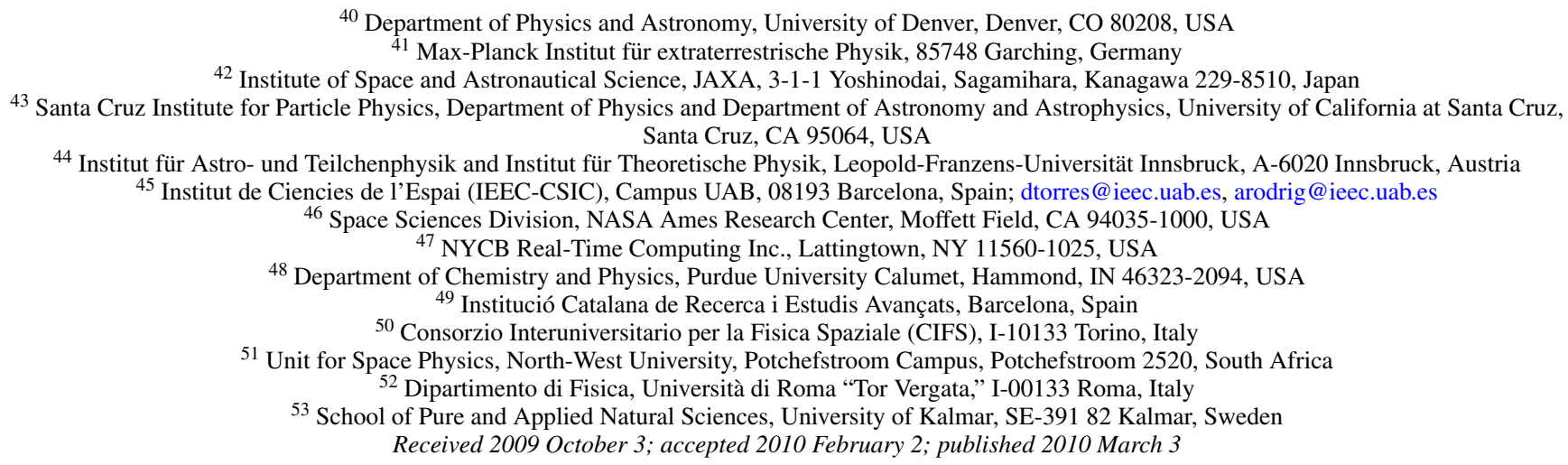

\section{ABSTRACT}

We report observation of the supernova remnant (SNR) IC $443($ G189.1+3.0) with the Fermi Gamma-ray Space Telescope Large Area Telescope (LAT) in the energy band between $200 \mathrm{MeV}$ and $50 \mathrm{GeV}$. IC 443 is a shell-type SNR with mixed morphology located off the outer Galactic plane where high-energy emission has been detected in the X-ray, GeV and $\mathrm{TeV}$ gamma-ray bands. Past observations suggest IC 443 has been interacting with surrounding interstellar matter. Proximity between dense shocked molecular clouds and $\mathrm{GeV}-\mathrm{TeV}$ gamma-ray emission regions detected by EGRET, MAGIC, and VERITAS suggests an interpretation that cosmic-ray (CR) particles are accelerated by the SNR. With the high gamma-ray statistics and broad energy coverage provided by the LAT, we accurately characterize the gamma-ray emission produced by the CRs accelerated at IC 443 . The emission region is extended in the energy band with $\theta_{68}=0.27 \pm 0.01$ (stat) \pm 0.03 (sys) for an assumed two-dimensional Gaussian profile and overlaps almost completely with the extended source region of VERITAS. Its centroid is displaced significantly from the known pulsar wind nebula (PWN) which suggests the PWN is not the major contributor in the present energy band. The observed spectrum changes its power-law slope continuously and continues smoothly to the MAGIC and VERITAS data points. The combined gamma-ray spectrum (200 MeV $<E<2 \mathrm{TeV}$ ) is reproduced well by decays of neutral pions produced by a broken power-law proton spectrum with a break around $70 \mathrm{GeV}$.

Key words: gamma rays: general - supernovae: individual (IC 443)

Online-only material: color figures

\section{INTRODUCTION}

IC 443 is a well-studied supernova remnant (SNR), possessing strong molecular line emission regions that make it a case for an SNR interacting with molecular clouds. The SNR is one of the best candidates for revealing the connection among SNRs, molecular clouds, and high-energy gamma-ray sources as reviewed by Torres et al. (2003).

IC 443 is located in the outer Galactic plane and listed as a core-collapse SNR, G189.1+3.0, in Green's catalog (Green 2004). The SNR has an angular extent of $\sim 45^{\prime}$ in the radio with a complex shape consisting of two half-shells with different radii (shells A and B; e.g., Fesen \& Kirshner 1980; Braun \& Strom 1986a, 1986b; Petre et al. 1988; Furst et al. 1990; Leahy 2004 , and references therein). Its age is uncertain: some analyses indicate a young age (3-4 kyr; e.g., Petre et al. 1988; Troja et al. 2008) but others indicate that it is older (20-30 kyr; e.g., Lozinskaya 1981; Chevalier 1999; Olbert et al. 2001; Gaensler et al. 2006; Bykov et al. 2008; Lee et al. 2008). Its distance has not been measured directly but is assumed to be $\sim 1.5 \mathrm{kpc}$, the distance to the Gem OB1 association to which the SNR belongs (e.g., Woltjer 1972; Olbert et al. 2001; Welsh \& Sallmen 2003; Gaensler et al. 2006). A pulsar wind nebula (PWN), CXOU J061705.3+222127, has been found in the southern periphery

\footnotetext{
${ }^{54}$ National Research Council Research Associate.

55 Royal Swedish Academy of Sciences Research Fellow, funded by a grant from the K. A. Wallenberg Foundation.
}

of the SNR but its association with the SNR has not yet been firmly established (Keohane et al. 1997; Olbert et al. 2001; Bocchino \& Bykov 2001; Leahy 2004; Gaensler et al. 2006; Troja et al. 2008). To this day pulsation has not been reported at the position of the putative pulsar.

A general picture has been drawn from past observations and analyses that a variety of dynamical processes are taking place in the complex structure of IC 443 (e.g., Lee et al. 2008; Troja et al. 2006, 2008, and references therein). The processes include: interaction of SNR shocks with molecular and atomic clouds of various densities which produced a break-out (shell B) from shell A as well as associated small-scale structures; interaction of the half-shells with another SNR G189.6+3.3 (e.g., Asaoka \& Aschenbach 1994; Keohane et al. 1997); penetration of shock fronts into dense molecular clouds leading to molecular line emission (e.g., Denoyer 1979a, 1979b; Denoyer \& Frerking 1981; Huang et al. 1986; Burton et al. 1988; van Dishoeck et al. 1993; Richter et al. 1995; Chevalier 1999; Hewitt et al. 2006); and interaction between the PWN and the environment (Olbert et al. 2001; Leahy 2004; Gaensler et al. 2006; Troja et al. 2008).

Of special interest for this study are the detections of high and very high energy (VHE) gamma rays in the IC 443 vicinity. EGRET detected a gamma-ray source above $100 \mathrm{MeV}$, cospatial ${ }^{56}$ with the SNR (3EG J0617+2238) (Sturner \& Dermer

\footnotetext{
56 We assume that the gamma-ray sources detected in the region are associated with locally accelerated cosmic rays (CRs) based on the spatial overlap with the IC 443 structure seen in the radio, IR, optical, and X-ray bands.
} 
1995; Esposito et al 1996; Lamb \& Macomb 1997; Hartman et al 1999). The MAGIC telescope discovered a VHE source, MAGIC J0616+225 (Albert et al. 2007) which is displaced with respect to the position of the EGRET source, and cospatial with what appears to be the most massive molecular cloud in the neighborhood detected in ${ }^{12} \mathrm{CO}$ and ${ }^{13} \mathrm{CO}$ emission lines (Burton et al. 1988; Dickman et al. 1992; Dame et al. 2001; Seta et al. 1998). VERITAS has confirmed the VHE emission (VER J0616.9+2230) and resolved the source to be extended (Acciari et al. 2009). The centroids of these three gamma-ray sources are displaced from that of the PWN.

The Large Area Telescope (LAT) data for IC 443 provide an exciting opportunity to study the interaction of an SNR with the interstellar medium, CR acceleration and subsequent injection to the Galactic space. The entire Milky Way has been deeply observed by the LAT, and modeling of the diffuse emission thereon allows the emission associated with IC 443 (the "IC 443 contribution") to be considered separately from the underlying Galactic diffuse emission, which has contributions from inverse Compton (IC) scattering of CR electrons (the "Galactic IC component") and CR electron and proton interactions with interstellar nuclei (the "Galactic CR contribution"). In the LAT data, the spatial extension of the contribution from IC 443 can be measured along with its broadband spectrum.

This paper is organized in the following sections. A brief description of the observation, event reconstruction, and gammaray selection is given in Section 2. The analysis procedure is explained in Section 3 including the instrument response function (IRF) and separation of the Galactic CR contribution, Galactic IC contribution, extragalactic emission, and instrumental background. We present results on the spatial extension and spectrum of the IC 443 contribution in Section 4. Discussion is given in Section 5, and the paper is concluded in Section 6.

\section{OBSERVATIONS, EVENT RECONSTRUCTION, AND GAMMA-RAY SELECTION}

\subsection{Observation in the Survey Mode}

The Fermi Gamma-ray Space Telescope, launched on 2008 June 11, has been surveying all sky with the LAT since 2008 August. Its wide field of view $(\sim 2.4 \mathrm{sr})$, large effective area $\left(\sim 8000 \mathrm{~cm}^{2}\right.$ at $\left.>1 \mathrm{GeV}\right)$, the improved point-spread function $(\mathrm{PSF})^{57}\left(\theta_{68}^{\mathrm{psf}} \sim 0.6 / 0.9\right.$ at $\left.E=1 \mathrm{GeV}\right)$ and the broad energy coverage $(20 \mathrm{MeV}-300 \mathrm{GeV})$ bring much improved sensitivity and gamma-ray statistics over its predecessor EGRET (Atwood et al. 2009).

The LAT is a pair-conversion telescope where a gamma ray is converted to an $e^{+} e^{-}$pair. Their trajectories are recorded in the tracker and the subsequent shower development is sampled both in the tracker and the calorimeter. The tracker is surrounded by a segmented anti-coincidence detector which is used to reject events induced by charged CRs (Atwood et al. 2009; Abdo et al. 2009a). The LAT PSF ( $\left.\theta_{68}^{\mathrm{psf}}\right)$ is determined at lower energies by multiple scattering in the tracker. At higher energies, the PSF approaches to the limit given by the granularity of the tracker

\footnotetext{
57 The PSF is significantly different for gamma rays detected in the front and back portions of the tracker described in this section: the first and second of the two values separated by "/" are for those detected in the front and back, respectively. We use $\theta_{68}$ and $\theta_{95}$ with superscripts $p s f$, error, and ext to quantify the PSF, source localization error, and source extension, respectively. The integrated probabilities in the two-dimensional (2D) angular radii of $\theta_{68}$ and $\theta_{95}$ are $68 \%$ and $95 \%$, respectively. For a symmetric 2D Gaussian distribution $\theta_{95}$ is $1.62 \times \theta_{68}$.
}

Table 1

Selection for the Data Set

\begin{tabular}{lc}
\hline \hline \multicolumn{1}{c}{ Parameter } & Value \\
\hline Time period (MET) & $239557417-268416079$ \\
Energy range & $200 \mathrm{MeV}-50 \mathrm{GeV}$ \\
ROI & $\leqslant 15^{\circ}$ in radius \\
Photon class & Pass 6 Diffuse \\
Additional cut & Zenith angle $\leqslant 105^{\circ}$ \\
\hline
\end{tabular}

channels: it is $0.16 / 0.26$ at $5 \mathrm{GeV}$ and $0.11 / 0.15$ at $10 \mathrm{GeV}$ (Atwood et al. 2009).

The LAT was operated in the nominal all-sky survey during the present observation. In the observation, the instrument axis was tilted from the zenith toward the orbit's north and south poles by $35^{\circ}$ or $39^{\circ}$ on alternate orbits to make sky coverage uniform. The trigger rate, mostly on CRs, was $\sim 2.2 \mathrm{kHz}$ in average and varied between the maximum of $\sim 5.0 \mathrm{kHz}$ and the minimum of $\sim 1.6 \mathrm{kHz}$ depending on the geomagnetic cutoff rigidity. Onboard filtering reduced the event rate to $\sim 450 \mathrm{~Hz}$ for the downlink. Data taking is disabled during passages through the South Atlantic Anomaly (Abdo et al. 2009a).

\subsection{Gamma-ray Selection}

Gamma-ray candidates are defined in three classes on the gamma-ray probability, background expected in orbit, current knowledge of the astronomical gamma-ray fluxes, and performance of the LAT. The Diffuse class has the tightest background rejection of the three (Atwood et al. 2009). However, the effective area becomes small and strongly dependent energy below $200 \mathrm{MeV}$. The averaged rate for the Diffuse class event was $\sim 0.6 \mathrm{~Hz}$ during the observation.

In the survey mode, the Earth limb, an extremely bright source of gamma rays, comes near the edge of the field of view. We have removed these gamma rays with the reconstructed zenith angles greater than $105^{\circ}$.

CR-induced background in the Diffuse class becomes comparable in intensity to gamma rays from the IC 443 region at energies below 100-200 MeV and above 50-100 GeV. The background consists of residual CRs misclassified as gamma rays and CRs that convert in the passive material just outside of the LAT without leaving a signal in the anti-coincidence detector. We limit the energy range of this analysis between $200 \mathrm{MeV}$ and $50 \mathrm{GeV}$ where the effective area and the instrumental background are best understood ${ }^{58}$.

The data analyzed here were obtained between 2008 August 4 and 2009 July 4. The gamma rays in the circular region of interest (ROI) of radius $15^{\circ}$ centered at the best-fit centroid of the IC 443 contribution to be determined in Section 4.1 $(\ell=189.05, b=3.03)$ are selected for later analyses. We refer to this set of events as the data set: the key selections described here are summarized in Table 1.

Events in the data set are binned in energy at 13 logarithmic steps of 0.184 starting from $200 \mathrm{MeV}$. The matching energydependent exposure is calculated based on the orbit location, pointing direction, orientation, and live-time accumulation of the LAT. The intensity is then calculated by dividing maps of counts with maps of exposure in each energy bin.

\footnotetext{
58 We are currently developing an improved event classification procedure to retain the higher effective area at lower energies and to reduce background contaminations in the entire energy range.
} 


\section{ANALYSIS PROCEDURE}

The present analysis focuses on determination of the centroid and extension of the IC 443 contribution after separation of the Galactic CR contribution in the region. The latter will reflect the detailed spatial structure of the molecular clouds through pionic and bremsstrahlung interactions, and potentially can be mistaken as a part of the IC 443 contribution. The uniform all-sky coverage of the LAT observation allows us to isolate the IC 443 contribution cleanly from all Galactic-scale contributions and to determine its extension accurately. The Sourcelike analysis has been designated specifically for this kind of analyses. Spectral analysis has been done with the LAT Science Tool gtlike $^{59}$ and has been cross-checked by Sourcelike. We give a brief description of the IRF and Sourcelike before proceeding to the fitting.

\subsection{Instrument Response Function}

The spatial extension and spectral features of the gamma-ray emission are studied by comparing the observation with predictions of source models. Predictions are made by convolving the spatial distribution and spectrum of the source models with the IRF and the exposure for the observation.

The IRF describes the overall performance of the instrument, event reconstruction, and gamma-ray selection. In Fermi LAT, it has been formulated, before the launch, using an instrument simulation program (Atwood et al. 2009). The simulation program has been calibrated against beam test results (Atwood et al. 2009; Abdo et al. 2009a) and the predicted IRF has been validated on several bright point sources in the early operation phase.

The variation in the trigger rate results in variation in the fraction of the trigger-enabled time (the "live-time fraction") between $\sim 94.3 \%$ and $\sim 81.5 \%$. Besides lowering the livetime fraction and the exposure, CR hits overlaid on a genuine gamma-ray track can reduce reconstruction efficiency and lead to incorrect event selection. The overall inefficiency has been found to scale linearly with the loss in the live-time fraction with a coefficient that depends on energy. The IRF used in the analysis, IRF P6_V3_Diffuse, has been corrected for inefficiency by, for example, $+23 \%,+16 \%$, and $+12 \%$ at $200 \mathrm{MeV}, 500 \mathrm{MeV}$, and $1 \mathrm{GeV}$, respectively.

\subsection{Extension Analysis with Sourcelike}

The intensity distribution observed by the LAT from the IC 443 region is shown in Figure 1 for a lower $(1-5 \mathrm{GeV})$ and a higher $(5-50 \mathrm{GeV})$ energy band for an area of $8^{\circ} \times 8^{\circ}$ centered at $(\ell, b)=\left(189^{\circ}, 3^{\circ}\right)$ with 0.1 pixelization. Spatial extension of the IC 443 contribution is determined on the intensity distribution using Sourcelike, an analysis tool developed by the LAT team. In the tool, likelihood fitting is iterated to the data set assuming spatial source models and a spatial background model: we use combination of a symmetric 2D Gaussian source model or a point-source model and the standard background model.

The standard background model used in Sourcelike is formulated by summing the Galactic CR contribution, Galactic IC contribution, and isotropic component ${ }^{60}$ given in the diffuse

\footnotetext{
59 Available from

http://fermi.gsfc.gov/ssc/data/access/lat/BackgroundModels.html,

the Fermi Science Support Center ur for the Science Tools.

60 The sum of the extragalactic background, unresolved sources, and instrumental background: its spatial distribution is assumed to be isotropic.
}
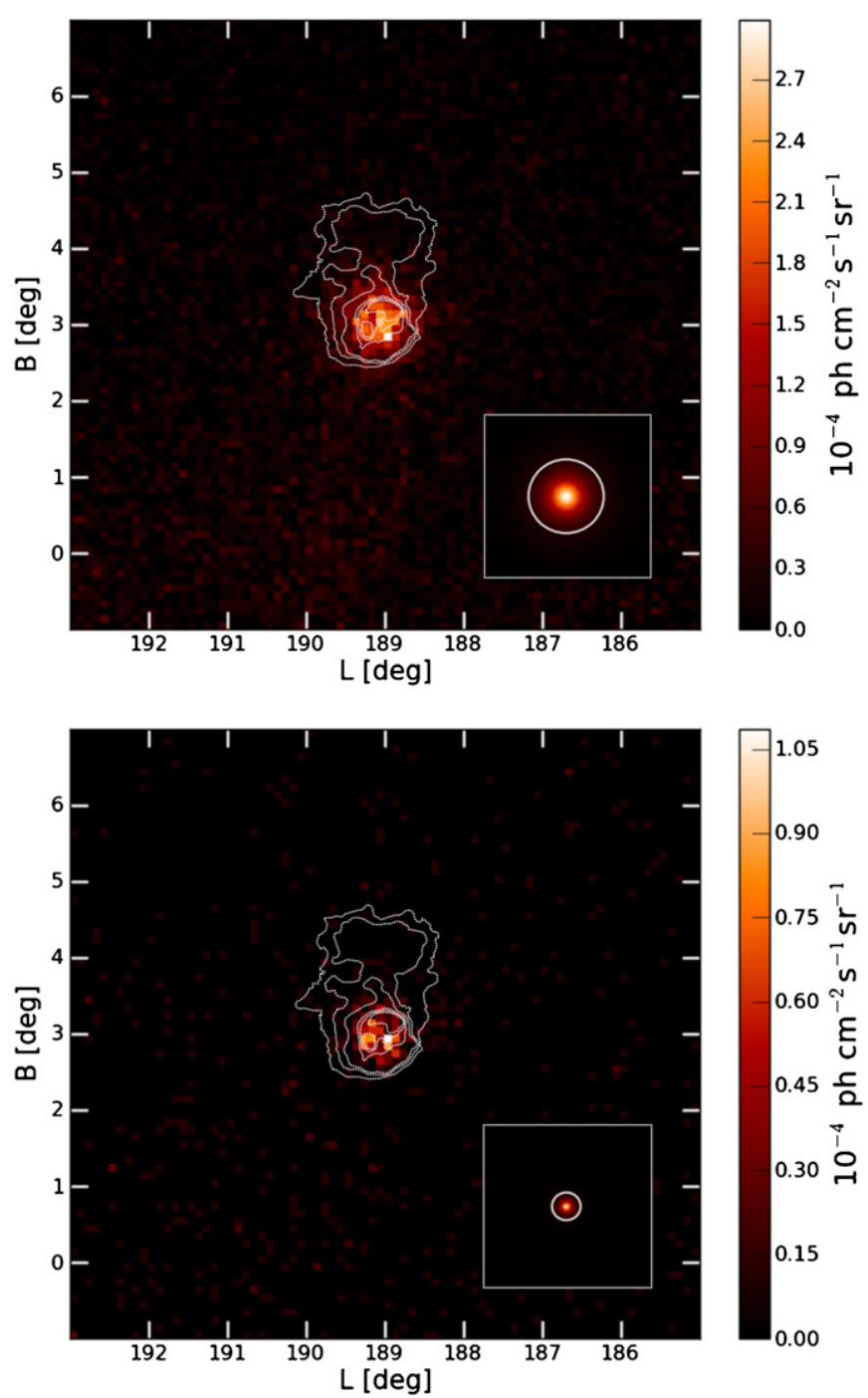

Figure 1. Intensity map of the IC 443 region in the $1-5 \mathrm{GeV}$ (left) and 5-50 GeV (right) bands. Units of intensity are $10^{-4} \mathrm{~cm}^{-2} \mathrm{~s}^{-1} \mathrm{sr}^{-1}$ for the color scale. The overlay is the $2.7 \mathrm{GHz}$ radio continuum contours taken from Furst et al. (1990). The insets are the spectrum-weighted LAT PSF for each energy band, with the white circles showing the corresponding $\theta_{68}^{\mathrm{psf}}$.

(A color version of this figure is available in the online journal.)

emission model ${ }^{61}$ : it is referred to as the background here after. All bright sources detected with the LAT (Abdo et al. 2009b) within $15^{\circ}$ of the centroid are included in the background.

The fit is performed for the entire data in the user-determined energy range. Absolute normalization of individual background components can be constrained or unconstrained in the fit: we leave the diffuse emission model as one unconstrained component and so are all bright sources in the ROI in the Sourcelike fit. The difference in test statistic (TS) values between the bestfit Gaussian distribution and the best-fit point source which is $2 \Delta \log ($ Likelihood) gives a measure of statistical significance of the extension. We refer to this difference as $\mathrm{TS}_{\mathrm{ext}}$ in this paper.

The ROI is energy dependent in the Sourcelike: $15^{\circ}$ at $200 \mathrm{MeV}$ and shrinks to a minimum of 3.5 at $50 \mathrm{GeV}$, which is at least a factor of 20 larger than $\theta_{68}^{\mathrm{psf}}$ of the LAT at the same energy

61 gll_iem_v02.fit and isotropic_iem_v02.txt available from the url given in footnote 59 . 
Table 2

Centroid and Extension of the IC 443 Contribution

\begin{tabular}{|c|c|c|c|c|c|c|}
\hline Model & $\ell\left(^{\circ}\right)$ & $b\left(^{\circ}\right)$ & $\theta_{68}^{\text {error }}\left({ }^{\circ}\right)^{a}$ & $\theta_{68}^{\text {ext }}\left(\left(^{\circ}\right)^{\mathrm{a}}\right.$ & $\theta_{95}^{\operatorname{ext}}\left(\left(^{\circ}\right)^{\mathrm{a}}\right.$ & $\mathrm{TS}_{\mathrm{ext}}{ }^{\mathrm{b}}$ \\
\hline \multicolumn{7}{|l|}{$1-5 \mathrm{GeV}$} \\
\hline Point source & 189.05 & 3.04 & 0.02 & $\ldots$ & $\ldots$ & 0 \\
\hline Gaussian & 189.05 & 3.05 & 0.02 & $0.27 \pm 0.03$ & $0.44 \pm 0.04$ & $+106 /+121$ \\
\hline \multicolumn{7}{|l|}{$5-50 \mathrm{GeV}$} \\
\hline Point source & 189.05 & 2.98 & 0.03 & $\ldots$ & $\ldots$ & 0 \\
\hline Gaussian & 189.06 & 3.00 & 0.03 & $0.26 \pm 0.04$ & $0.42 \pm 0.07$ & $+81 /+212$ \\
\hline \multicolumn{7}{|l|}{$1-50 \mathrm{GeV}$} \\
\hline Point source & 189.05 & 3.02 & 0.02 & $\ldots$ & $\ldots$ & 0 \\
\hline Gaussian & 189.05 & 3.03 & 0.02 & $0.27 \pm 0.03$ & $0.45 \pm 0.05$ & $+212 /+362$ \\
\hline
\end{tabular}

Notes.

${ }^{\text {a }}$ Errors of centroids and extensions quoted include systematic errors.

b The two values shown in this column are the $\mathrm{TS}_{\text {ext }}$ obtained with the default PSF (the first) and worst-case PSF (the second) described in the text.

and more than a factor of 10 larger than the spatial extension $\left(\theta_{68}^{\text {ext }}\right)$ of the source to be determined later.

\section{THE IC 443 CONTRIBUTION}

\subsection{Spatial Extension of the IC 443 Contribution}

Two energy bands, $1 \mathrm{GeV}<E<5 \mathrm{GeV}$ (the lower energy band) and $5 \mathrm{GeV}<E<50 \mathrm{GeV}$ (the higher energy band), have been selected to study the spatial extension of the IC 443 contribution. Sourcelike has been run for events in the two energy bands separately as well as in the combined energy band under a 2D Gaussian and point-source hypotheses. The best-fit results are summarized in Table 2.

The number of gamma rays in the fitted Gaussian distribution is 4972 for $200 \mathrm{MeV}<E<1 \mathrm{GeV}, 1597$ for $1 \mathrm{GeV}<$ $E<5 \mathrm{GeV}$, and 236 for $5 \mathrm{GeV}<E<50 \mathrm{GeV}$. For a given PSF, the accuracy of centroid determination is predicted to improve proportionally to the inverse of the square root of the number of events. The accuracy quoted in Table 2 is consistent with this prediction for the effective PSF averaged over events in the energy bands $1 \mathrm{GeV}<E<5 \mathrm{GeV}$ and $5 \mathrm{GeV}<E<50 \mathrm{GeV}$.

The difference in TS $\left(\mathrm{TS}_{\mathrm{ext}}\right)$ between the symmetric $2 \mathrm{D}$ Gaussian and point hypotheses is +106 to $+121(10.3 \sigma-11.0 \sigma)^{62}$ for the $1-5 \mathrm{GeV}$ band and +212 to $+81(14.6 \sigma-9.0 \sigma)$ for the $5-50 \mathrm{GeV}$ band. The centroids for the two bands are consistent within 0.04 . The IC 443 contribution is extended to $\theta_{68}^{\text {ext }}=0.26-0.27$ in the two energy bands. The centroid in the high-energy band is displaced southward by $\sim 0.04(\sim 1.5 \sigma)$ in the Galactic coordinate from that in the low-energy band.

The results on source location and extension are robust: TS values have been examined at discrete points offset from the best-fit location and extension to confirm the fit. To verify the fit further, we have generated 100 simulated sets of events assuming the best-fit centroid, extension, and background with the LAT Science Tool gtobssim. The simulated data are then processed through Sourcelike under 2D Gaussian and pointsource hypotheses. The distribution of $\mathrm{TS}_{\text {ext }}$ between the two hypotheses is consistent with the values given in Table 2 .

The point-source hypothesis is rejected at $\mathrm{TS}_{\mathrm{ext}}>81$ or $>9 \sigma$ independently in the two energy bands and at $\mathrm{TS}_{\mathrm{ext}}>212$ or $>14 \sigma$ in the combined energy band. The extensions in the two

\footnotetext{
62 The two $\mathrm{TS}_{\mathrm{ext}}$ values quoted are as follows: the first one for that used in P6_V3_diffuse; and the second one for a worst-case PSF to be used later in Section 5 to obtain a conservative systematic error.
}

energy bands are mutually consistent within the errors given in Table 2.

The radial profiles of event distribution around the centroid are shown in Figure 2 for the low- and high-energy bands together with the profile predicted for the point-source hypothesis, which is the LAT PSF weighted with the spectral distribution of the events Sourcelike associated with the source under the point-source hypothesis.

Extension was poorly determined for $E=200 \mathrm{MeV}-1 \mathrm{GeV}$ because of the large PSF of the LAT in the energy range (Atwood et al. 2009). However, the centroid and extension are consistent with the extension determined above $1 \mathrm{GeV}$ and given in Table 2. Hence, we assume the same 2D Gaussian distribution in the entire energy range.

\subsection{Spectrum of the IC 443 Contribution}

The spectrum of the IC 443 contribution is fitted by the Science Tool gtlike, the Fermi standard tool, as well as by Sourcelike. In gtlike, we have to assume a spatial template for all spectral components included in the fitting. The data set is assumed to be a sum of three contributions: the bestfit 2D Gaussian distribution given for the $E=1-50 \mathrm{GeV}$ range in Table 2 which represents the IC 443 contribution $^{63}$; the background whose spatial distribution is represented by the sum of gll_iem_v02.fit and isotropic_iem_v02.txt; and the bright sources listed in Abdo et al. (2009b) in the square region of $8^{\circ} \times 8^{\circ}$ centered at the best-fit centroid $(\ell=189.05, b=3.03)$.

The fitted IC 443 spectra from gtlike and Sourcelike agree well within the total error. We adopt the spectrum obtained with gtlike and tabulate in Table 3. It is converted to spectral energy density (SED) and shown by circles with error bars in Figure 3. Squares with error bars in the figure are the background spectrum normalized to the solid angle subtended by $\theta_{95}^{\text {ext }}=0.45$ around the centroid given in Table 2 . The IC 443 contribution is approximately 20 times higher than the background in the entire energy band.

The SED of the IC 443 contribution thus determined has been fitted with single power-law (PL) and broken power-law models: the results are tabulated in Table 4. The single power-law fit fails to represent the spectrum giving a large reduced chi-square of $\sim 9$ while the broken power-law fit represents the overall shape quite well giving a small reduced chi-square $(\sim 1.0)$ as shown

\footnotetext{
63 The extension could not be determined at a high statistical significance for $E=200 \mathrm{MeV}-1 \mathrm{GeV}$ but the spatial distribution of gamma rays is consistent with those given in Table 2 .
} 

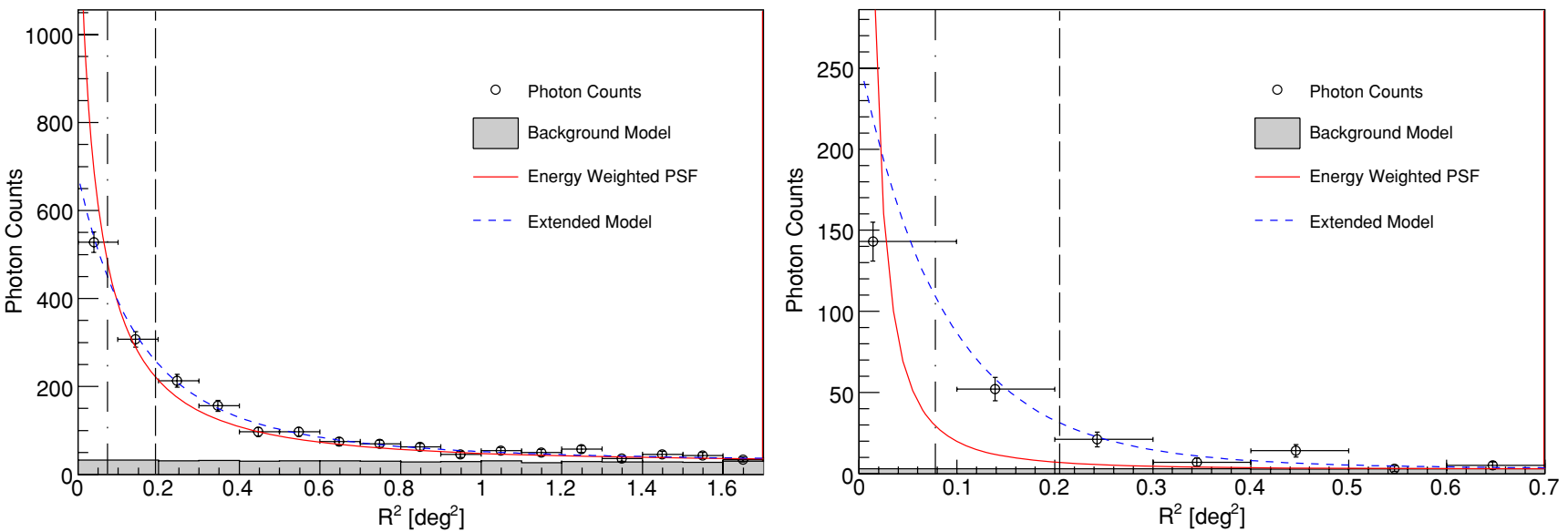

Figure 2. Radial profile of the gamma rays SourceLike associated with the IC 443 contribution (data points), compared with the effective spectrum-weighted LAT PSF (solid red line) and the fitted 2D Gaussian extended model (dashed blue line). The left and right panels correspond to the low (1-5 GeV) and high (5-50 GeV) energy bands, respectively. The points in the count profile are plotted at the weighted average radial positions within their respective bins. The vertical dash-dot and dashed lines correspond to the fitted extension $\theta_{68}^{\text {ext }}$ and $\theta_{95}^{\text {ext }}$ given in Table 2 for the respective energy bands.

(A color version of this figure is available in the online journal.)

Table 3

Spectrum of the IC 443 Contribution

\begin{tabular}{rccc}
\hline \hline$E_{\text {center }}(\mathrm{MeV})$ & $d N / d E\left(\mathrm{~cm}^{-2} \mathrm{~s}^{-1} \mathrm{MeV}^{-1}\right)$ & $d N / d E($ Stat Error) & $d N / d E$ (Sys Error) \\
\hline 247.31 & $8.63 \times 10^{-10}$ & $4.80 \times 10^{-11}$ & $6.37 \times 10^{-11}$ \\
378.19 & $4.11 \times 10^{-10}$ & $1.87 \times 10^{-11}$ & $2.52 \times 10^{-11}$ \\
578.32 & $1.72 \times 10^{-10}$ & $7.81 \times 10^{-12}$ & $8.89 \times 10^{-12}$ \\
884.36 & $8.31 \times 10^{-11}$ & $3.59 \times 10^{-12}$ & $6.11 \times 10^{-12}$ \\
1352.34 & $3.25 \times 10^{-11}$ & $1.65 \times 10^{-12}$ & $3.12 \times 10^{-12}$ \\
2067.96 & $1.51 \times 10^{-11}$ & $8.36 \times 10^{-13}$ & $1.78 \times 10^{-12}$ \\
3162.27 & $5.81 \times 10^{-12}$ & $4.06 \times 10^{-13}$ & $8.14 \times 10^{-13}$ \\
4835.67 & $2.40 \times 10^{-12}$ & $2.05 \times 10^{-13}$ & $3.90 \times 10^{-13}$ \\
7394.58 & $7.49 \times 10^{-13}$ & $8.98 \times 10^{-14}$ & $1.38 \times 10^{-13}$ \\
11307.60 & $2.80 \times 10^{-13}$ & $4.29 \times 10^{-14}$ & $5.61 \times 10^{-14}$ \\
17291.30 & $9.13 \times 10^{-14}$ & $1.93 \times 10^{-14}$ & $1.82 \times 10^{-14}$ \\
26441.41 & $2.95 \times 10^{-14}$ & $8.94 \times 10^{-15}$ & $5.90 \times 10^{-15}$ \\
40433.51 & $6.46 \times 10^{-15}$ & $3.35 \times 10^{-15}$ & $1.29 \times 10^{-15}$ \\
\hline
\end{tabular}

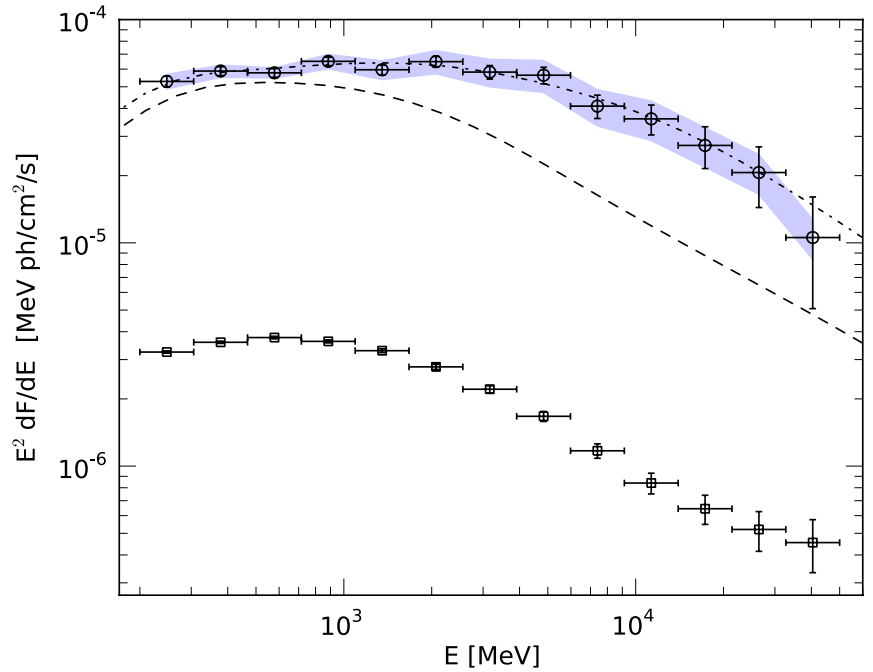

Figure 3. Gamma-ray spectrum of the IC 443 contribution: the upper and lower data points represent the IC 443 contribution and the total background, respectively. The background has been scaled to match the solid angle subtended by a disk of radius $\theta_{95}^{\text {ext }}=0.45$. Errors are shown by the bars (statistical) and the gray band (systematic). The lines represent the pionic gamma-ray spectra produced by the Galactic CR proton at IC 443 scaled up by a factor of 100 (dashed) and by the locally accelerated proton population with the best-fit broken power-law spectrum described in the text (dot-dash).

(A color version of this figure is available in the online journal.) in Table 4. The SED of the IC 443 contribution is plotted with those from previous observations, EGRET (Esposito et al 1996), MAGIC (Albert et al. 2007), and VERITAS(Acciari et al. 2009) in Figure 4.

\subsection{Systematic Error in Determination of the Centroid, Extension, and Spectrum}

When we determine the centroid of the IC443 contribution, uncertainty in the spatial distribution of the Galactic diffuse emission adds to the systematic error. The spatial template is taken from the standard diffuse emission model, gll_iem_v02.fit. To confirm our analysis, we have fitted the data set with the standard version of GALPROP for Fermi LAT (GALDEF 54_59Xvarh8S; Strong \& Moskalenko 1998; Strong et al. 2001, 2009) made of the CO line survey by Dame et al. (2001) and $\mathrm{H}$ I survey by Kalberla et al. (2005) as well as with a gas model made of the $A_{V}$ map by Dobashi et al. (2005) and H I survey by Kalberla et al. (2005). The two alternate gas models have given centroids consistent with that given in Table 2.

The residual misalignment of the LAT and the star tracker can also contribute to the systematic error: the source localization has been verified on orbit using bright point sources to \pm 30 arcsec as of 2009 August. The combination of all errors described here gives the overall systematic localization error in Table 5. 
Table 4

Spectral Fit to the IC 443 Contribution

\begin{tabular}{lcccccc}
\hline \hline \multicolumn{2}{c}{ Model } & $\gamma_{1}$ & $\gamma_{2}$ & $E_{\text {break }}(\mathrm{GeV})$ & $F_{200}{ }^{\mathrm{a}}\left(10^{-7} \mathrm{~cm}^{-2} \mathrm{~s}^{-1}\right)$ & $\chi^{2} / \mathrm{dof}$ \\
\hline IC 443 & Broken PL & $1.93 \pm 0.03$ & $2.56 \pm 0.11$ & $3.25 \pm 0.6$ & $2.85 \pm 0.07$ & $8.9 / 9$ \\
IC 443 & PL & $2.08 \pm 0.02$ & $\cdots$ & $\cdots$ & $3.00 \pm 0.07$ & $90 / 11$ \\
\hline
\end{tabular}

Note. ${ }^{\text {a }}$ Total flux integrated above $200 \mathrm{MeV}$ obtained with gtlike assuming the best-fit broken powerlaw model and the best-fit 2D Gaussian spatial distribution.

Table 5

Summary of Locations and Extensions of the Gamma-ray Sources

\begin{tabular}{lcccc}
\hline \hline Observation & $\ell\left(^{\circ}\right)$ & $b\left(^{\circ}\right)$ & $\theta_{68}^{\text {error }}$ of Localization $\left(^{\circ}\right)$ & $\theta_{68}^{\text {ext }}$ of Extension $\left(^{\circ}\right)$ \\
\hline EGRET & 189.00 & 3.05 & $0.13\left(\theta_{95}^{\text {error }}\right)$ & N/A \\
MAGIC & 189.03 & 2.90 & \pm 0.025 (stat) \pm 0.017 (sys) & N/A \\
VERITAS & 189.07 & 2.92 & \pm 0.03 (stat) \pm 0.08 (sys) & $0.24 \pm 0.05$ (stat) \pm 0.06 (sys) \\
Fermi & 189.05 & 3.03 & \pm 0.01 (stat) \pm 0.02 (sys) & $0.27 \pm 0.01$ (stat) \pm 0.03 (sys)
\end{tabular}

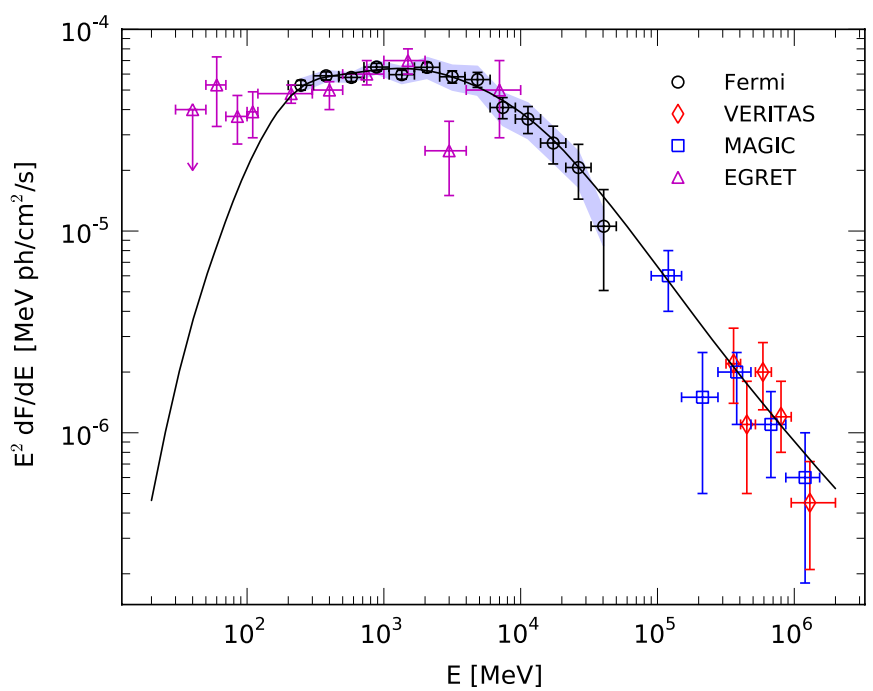

Figure 4. Broadband SED of the four sources: EGRET (purple triangles), MAGIC (blue squares), VERITAS (red diamonds), and Fermi (black circles). The solid line is the same as the dot-dash line in Figure 3. The systematic and statistical errors of the Fermi data points are also the same as in Figure 3.

(A color version of this figure is available in the online journal.)

Our flux measurement depends on the knowledge on the effective area as a function of gamma-ray energy. We estimate the systematic error in the effective area to be $10 \%, 5 \%$, and $20 \%$ at $E=100 \mathrm{MeV}, 562 \mathrm{MeV}$, and $>10 \mathrm{GeV}$, respectively.

Uncertainty in the background used in gtlike and Sourcelike can also introduce error in the flux measurement. This uncertainty is estimated to be $\sim 20 \%$ for $200 \mathrm{MeV}-1 \mathrm{GeV}$ and $\sim 30 \%$ for $>1 \mathrm{GeV}$ of the background (Abdo et al. 2009c, 2009d). Systematic error at each energy bin is determined through a linear interpolation in $\log _{10}(E)$ among the values quoted above.

The PSF used in this analysis has been derived on the detector simulation which was itself verified in accelerator tests (Atwood et al. 2009). As gamma-ray statistics improves, the PSF will be updated against measurement on bright point sources. In the present study, we have used a preliminary upper limit to assess possible systematic error introduced by inaccurate formulation of PSF: we consider this as the "worst-case" PSF. The worstcase PSF $\left(\theta_{68}^{\text {psf }}\right)$ gives a widest limit while the for $E>5 \mathrm{GeV}$ is about $40 \%$ larger than the default PSF. The source centroid comes out to be consistent within the total error when Sourcelike is run with the worst-case PSF. We have included the difference in the systematic error given in Tables 2 and 5.

\section{DISCUSSION}

The IC 443 system consists of a complex distribution of molecular and atomic clouds in the southern rim of shell A (e.g., Snell et al. 2005; Lee et al. 2008, and references therein). Molecular clouds wrap around the southern rim and the boundary region between shells A and B (Beichman et al. 1988; Seta et al. 1998; Dickman et al. 1992; Dame et al. 2001). Molecular lines from shocked gas have been found in several clouds suggesting interaction with the blast wave at multiple sites (e.g., Cornett et al. 1977; Denoyer \& Frerking 1981; Huang et al. 1986; Burton et al. 1988; Dickman et al. 1992; van Dishoeck et al. 1993; Richter et al. 1995; Seta et al. 1998; Snell et al. 2005). A prominent band of H I gas has also been found in the southeastern part of shell A (Denoyer 1978; Giovanelli \& Haynes 1979; Lee et al. 2008, and references therein.). Some parts of the H I gas are found to be shocked (Braun \& Strom 1986a; Lee et al. 2008).

The total mass of the molecular gas in the region is estimated to be $\sim 1 \times 10^{4} M_{\odot}$ (Torres et al. 2003), of which only a small fraction is shocked (Snell et al. 2005). The total mass in the H I belt is estimated at $\sim 730 M_{\odot}$ of which $\sim 500 M_{\odot}$ is shocked (Lee et al. 2008). Despite past extensive observations and analyses, little is known about how the multiple shell-like structures are spatially correlated and where one or more supernova explosions took place.

In the group of shocked molecular clouds schematically shown in Figure 5, Cloud $\mathrm{G}^{64}$ lies closest to the centroid of the MAGIC and VERITAS sources (Huang et al. 1986). It appears to be extended by $\sim 8^{\prime}$ and overlaps with a non-shocked $C O$ cloud formation (Huang et al. 1986; Burton et al. 1988; Dickman et al. 1992). Chevalier (1999) has suggested that interaction between shell $\mathrm{A}$ and Cloud $\mathrm{G}$ is responsible for the gamma-ray emission observed by EGRET. Existence of an $\mathrm{OH}$ maser in the cloud suggests that the densities reach $\sim 10^{4} \mathrm{~cm}^{-3}$ (Frail et al. 1996; Hewitt et al. 2006, 2008). A later CO line observation by Snell et al. (2005) found a compact core of extension $\sim 1^{\prime}-2^{\prime}$ in Cloud $\mathrm{G}$ at $(\ell=189.03, b=2.90)$. Fainter maser emission has also been found in Clouds B and D at $(\ell=189.18, b=2.97)$ and $(\ell=189.25, b=3.13$ ), respectively (Hewitt et al. 2006, 2008).

The locations and extensions of the gamma-ray emission from IC 443 detected by EGRET (Hartman et al 1999), MAGIC (Albert et al. 2007), VERITAS (Acciari et al. 2009), and Fermi LAT are summarized in Table 5 and shown in Figure 5. Fermi

\footnotetext{
${ }^{64}$ Labeling is given by Denoyer (1979b) and Huang et al. (1986).
} 


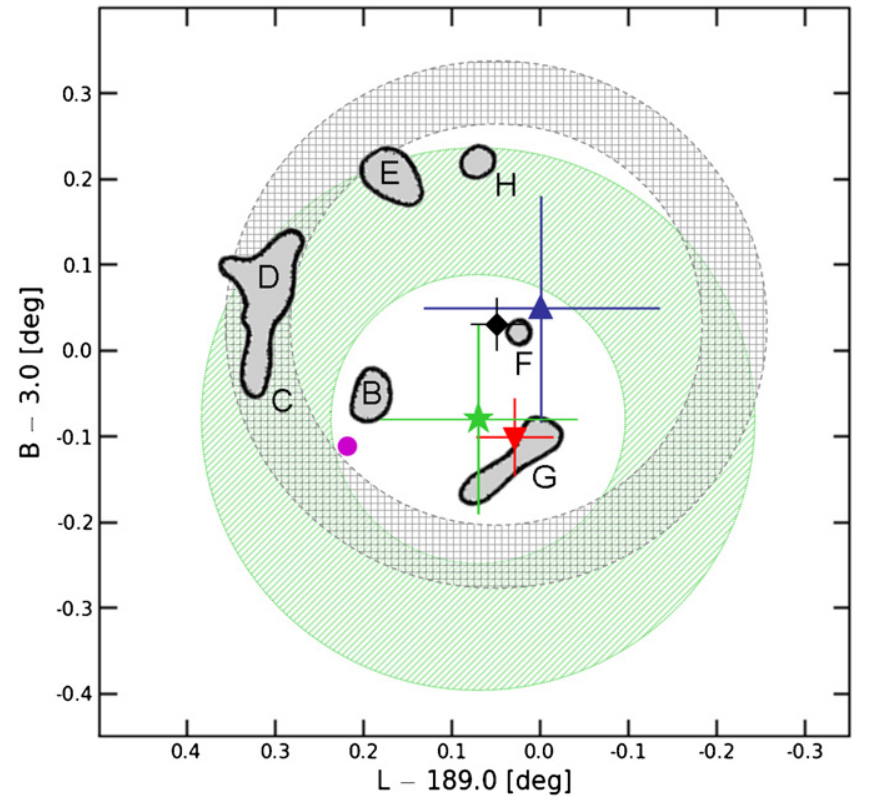

Figure 5. Locations and extensions of the four gamma-ray sources: EGRET centroid $(\triangle), M A G I C$ centroid $(\nabla)$, VERITAS centroid (star), and Fermi LAT centroid $(\diamond)$. The respective localization errors as tabulated in Table 5 are shown as crosses. Best-fit spatial extensions of the Fermi (cross-hatched band) and VERITAS (striped green band) sources are drawn as rings with radii of $\theta_{68}^{\text {ext }}$ and widths of $\pm 1 \sigma$ error. The PWN location is shown as a dot. Contours are the locations and shapes of the local shocked molecular clouds taken from Huang et al. (1986)

(A color version of this figure is available in the online journal.)

LAT gives the best source localization or the smallest error circle $\left(\theta_{68}^{\text {error }}\right)$ for the $2 \mathrm{D}$ Gaussian centroid $(0.03)$ and a precise determination of the source extension $(0.27 \pm 0.01$ (stat) \pm 0.03 (sys)). Our centroid determined for $1 \mathrm{GeV}<E<50 \mathrm{GeV}$ $\left(\ell=189^{\circ} .05, b=3.03\right)$ is 0.05 away from the EGRET source $(3 E G \mathrm{~J} 0617+2238)$ but within $\theta_{95}^{\text {error }}$ of their localization error; 0.15 from the MAGIC source $(\mathrm{J} 0610+225)$ which is at more than 5 times their localization error $\left(\theta_{68}^{\text {error }}\right)$; and 0.12 from the VERITAS source (VER J0616.9+2230) or at 1.5 times their localization error $\left(\theta_{68}^{\text {error }}\right)$.

The measured source extension, $\theta_{68}^{\text {ext }}=0.27 \pm 0.01$ (stat) \pm 0.03 (sys), is comparable with $\theta_{68}^{\text {ext }}=0.24 \pm 0.05$ (stat) \pm 0.06 (sys) ${ }^{65}$ given by VERITAS. The two extended regions overlap almost completely. The three shocked clouds with the $\mathrm{OH}$ maser (Clouds B, D, and G) are within our measured extension and so are other shocked clouds (Clouds C, E, F, and $\mathrm{H})$. The PWN localized at $(\ell=189.227, b=2.897)$ by Olbert et al. (2001) and Gaensler et al. (2006) is 0.26 away from our centroid but within our measured extension $\theta_{68}^{\text {ext }}$.

The Fermi spectrum of the IC 443 contribution shown in Figure 3 is flat between a few $100 \mathrm{MeV}$ and $\sim 3 \mathrm{GeV}$, suggesting the origin being mostly neutral pions produced by protons. ${ }^{66}$ The dashed line in the figure represents the gamma-ray spectrum expected from a $10^{4} M_{\odot}$ cloud bombarded with the Galactic CR protons predicted at IC 443 scaled up by a factor of 100 . The

\footnotetext{
65 We assume the extension is modeled by a symmetric 2D Gaussian and converted to $\theta_{68}=1.51 \times \theta_{1 \sigma}$.

66 We include CRs and target nuclei heavier than the proton (the alpha particle and heavier nuclei) in "protons" throughout this paper. In the approximation we adopt here (Gaisser \& Schaefer 1992), these can be accounted for by multiplying a "nuclear factor" $(\sim 1.7)$ without changing the $C R$ proton spectrum. The known gamma-ray producing particle processes which do not go through neutral pions (e.g., $\eta^{0} \rightarrow \gamma \gamma$ and direct photon production) contribute less than $1 \%$ in the present energy range.
}

Galactic CR spectrum is taken from the standard GALPROP (54_59Xvarh8S) (Strong \& Moskalenko 1998; Strong et al. 2001, 2009) and the parameterized cross section for $p p \rightarrow \gamma$ by Kamae et al. (2006). In GALPROP, the Galactic CR proton spectrum depends on the radius from the Galactic center and the displacement from the Galactic plane. The spectrum at the radius of IC 443 is $\sim 10 \%$ lower than that in the solar vicinity and has a power-law shape with index $\sim 2.7$.

The Fermi SED is compared with those of EGRET, MAGIC, and VERITAS in Figure 4. The EGRET spectrum is consistent with our spectrum except for their $3 \mathrm{GeV}$ point. MAGIC and VERITAS do not overlap with the LAT in the energy coverage. Their fluxes are consistent with ours if extrapolated down to $\sim 50 \mathrm{GeV}$ by assuming their measured power-law indices.

Since the source regions of Fermi LAT and VERITAS overlap within their respective uncertainties listed in Table 5, we can judiciously proceed to fit the two spectra with one spectral model.

On the assumption that the distance is $d=1.5 \mathrm{kpc}$, the isotropic luminosity of IC 443 integrated over the energy band $(0.2-50 \mathrm{GeV})$ is $1.2 \times 10^{35} \mathrm{erg} \mathrm{s}^{-1}$. Electron bremsstrahlung can hardly explain the observed IC 443 gamma-ray emissivity: the cross sections for bremsstrahlung and pionic gammaray emission are similar in the present energy band, so the bremsstrahlung-to-pion ratio is approximately the ratio of $\mathrm{CR}$ electron and proton fluxes which is $\sim 0.01$. The observed gamma-ray flux is too high for bremsstrahlung to be the dominant process. Inverse Compton scattering cannot explain the observed IC 443 gamma-ray emission either: the gas density of the emission region is $\sim 50-100 \mathrm{~cm}^{-3}$ and the Compton-tobremsstrahlung ratio is $\sim 0.01-0.001$ for the seed photon density of the cosmic microwave background. We note that there is no bright source of seed photons known in the region of the IC 443 contribution. The gamma-ray energy will be strongly bound by the electron spectrum which likely rolls down similarly as the proton spectrum. Our observation, however, does not rule out a small contribution from bremsstrahlung near the minimum of the present energy band.

In a hadronic scenario, the observed photon spectrum up to $\mathrm{TeV}$ energies can be well fitted by an underlying pion-producing proton population with a broken power-law spectrum $F_{p}\left(T_{p}\right)=$ $5.9 \times 10^{-2}\left(T_{p} / 69 \mathrm{GeV}\right)^{-\alpha}\left(10^{4} M_{\odot} / M_{\mathrm{gas}}\right) \mathrm{cm}^{-2} \mathrm{~s}^{-1} \mathrm{GeV}^{-1}$, where $\alpha$ is $2.09 \pm 0.04$ for $T_{p}<69 \mathrm{GeV}$ and $2.87 \pm 0.07$ for $T_{p}>69 \mathrm{GeV}$, respectively, and $M_{\mathrm{gas}}$ is the gas mass in the interaction region. The error (statistical) in the fitted break energy is $\pm 25 \mathrm{GeV}$ and the chi-square for the best-fit broken powerlaw model is 9.6/14 per degree of freedom. Assuming the gas density $\left(n \mathrm{~cm}^{-3}\right)$ is uniform and the proton spectrum is the broken power-law everywhere in the interaction region, the total energy of the interacting protons is given by $W_{p}(>0.5 \mathrm{GeV})=$ $5.6 \times 10^{48}\left(n / 240 \mathrm{~cm}^{-3}\right)^{-1}(d / 1.5 \mathrm{kpc})^{2}$ erg. Note that the pion production threshold is $\sim 0.5 \mathrm{GeV}$. Taking $M_{\text {gas }} \sim$ $10^{4} M_{\odot}$ and a gas volume ranging from $4 \pi / 3 \times\left(\theta_{68}^{\text {ext }} d\right)^{3}=5 \times$ $10^{58} \mathrm{~cm}^{3}$ up to $4 \pi / 3 \times\left(\theta_{95}^{\text {ext }} d\right)^{3}=2 \times 10^{59} \mathrm{~cm}^{3}$, we obtain $n \sim 60-240 \mathrm{~cm}^{-3}$, giving $W_{p}(>0.5 \mathrm{GeV})=(0.56-2.2) \times$ $10^{49}$ erg. We note that energies carried by local nuclear CRs outside of the interaction region and by local leptonic CRs are not included in the estimation. The fitted gamma-ray spectrum is shown in Figures 3 and 4. We note that inclusion of the MAGIC points in the fit does not change the above results.

Broadband gamma-ray spectral models have been proposed assuming CR interaction with interstellar gas in IC 443 by 
Torres et al. (2008) and Zhang \& Fang (2008). Torres et al. (2008) model the CR diffusion in the SNR to allow spectral differences in the protons interacting with the ambient gas in the shell and in a detached molecular cloud. Zhang \& Fang (2008) predict one contribution to come from the SNR shell evolving in the interstellar matter and the other from CR interaction with molecular clouds. One difference between the two models is that Zhang \& Fang (2008) include inverse Compton scattering as a possible emission mechanism.

The combined spectrum of Fermi and VERITAS gives a strong constraint to spectral models for the IC 443 contribution. Since the spectrum of the dominant CR component (proton) is rolling over at $\sim 70 \mathrm{GeV}$, secondary electrons and positrons can only contribute at energies $E_{\gamma}<7 \mathrm{GeV}$. This constrains the parameter space of the model by Zhang \& Fang (2008). The overlap between the Fermi and VERITAS spatial extensions and the smooth spectral transition from Fermi to VERITAS constrain the parameter spaces of the models by Torres et al. (2008) and Zhang \& Fang (2008).

We discuss briefly about possible mechanisms behind the broken power-law form of the proton spectrum deduced from the gamma-ray observation of IC 443. The most obvious one is escape of highest energy CRs from the acceleration site. When accelerated $\mathrm{CR}$ protons exceed the maximum energy determined by the magnetic field and linear scale of the acceleration site, they escape into the Galactic space. Theory of diffusive shock acceleration (DSA) assumes spherically symmetric morphology and predicts the CR spectrum to roll over exponentially at the maximum energy. The maximum energy depends on the condition of the acceleration site: Ptuskin \& Zirakashvili (2005) have incorporated various instabilities into DSA and predict the maximum energy as a function of the SNR age. For the age of IC 443 ( $\sim 30 \mathrm{kyr}$ ), the maximum proton energy can be around $100 \mathrm{GeV}$ (Figure 1 of Ptuskin \& Zirakashvili 2005) or near the observed break energy $(\sim 69 \mathrm{GeV})$ beyond which the spectrum is assumed to cutoff exponentially. We have fitted the observed gamma-ray spectrum with a single power law, exponentially cutoff proton spectrum to get chi-square per degree of freedom of 30.3/15 much higher than 9.6/14 for the broken powerlaw spectrum. This simple statistical test therefore suggests that the observed broadband gamma-ray spectrum is inconsistent with the simple DSA-based CR escape scenario which predicts a simple power law with an exponential cutoff in the proton spectrum. We also note that DSA has mostly been studied for uniform gas densities around $1 \mathrm{~cm}^{-3}$, while the gas around IC 443 is inhomogeneous and in various shocked molecular clouds around the remnant is also denser.

Historically, after the discoveries of the EGRET source (Sturner \& Dermer 1995; Esposito et al 1996) and the hard X-ray source (Keohane et al. 1997) but before the discoveries of the PWN (Olbert et al. 2001) and MAGIC source (Albert et al. 2007), models have been proposed to explain the emission between $\sim 5 \mathrm{keV}$ and $\sim 5 \mathrm{GeV}$ by bremsstrahlung in dense clouds (e.g., Bykov et al. 2000) with possible mix of synchrotron (e.g., Sturner et al. 1997). The SNR was also studied as a part of nonlinear shock evolution in various environments (e.g., Baring et al. 1999). While their predictions for IC 443 are not supported by the later observations including the present one, parameters in these studies can be readjusted to describe the bremsstrahlung contribution discussed below.

The bremsstrahlung likely makes a non-negligible contribution below $E_{\gamma}=200 \mathrm{MeV}$ where the EGRET data points exceed the best-fit pionic spectrum (see Figure 4). As our un- derstanding of the IRF and CR-induced background improves, analysis will be extended to energies lower than $200 \mathrm{MeV}$ and the bremsstrahlung spectrum component will be determined accurately. The hard X-ray SED measured by Beppo-SAX (Bocchino \& Bykov 2000) is substantially higher than that by XMM-Newton (Bocchino \& Bykov 2001), which may suggest bremsstrahlung contribution near the PWN location as has been discussed by Sturner et al. (1997) and Bykov et al. (2000).

\section{CONCLUSIONS}

We have studied gamma-ray emission from the nearby SNR IC 443 (G189.1+3.0) using the first 11 months of science data from the Fermi LAT. The uniform sky coverage and high gamma-ray statistics of the observation have enabled us to separate the genuine IC 443 contribution from the emissions by Galactic CRs on interstellar gas, inverse Compton scattering by Galactic CR electrons on the large-scale interstellar radiation field, extragalactic sources, and instrumental background.

Based on the extension study described in Section 4.1 and the spectral analysis described in Section 4.2 as well as discussions given in Section 5, we conclude the following.

1. The gamma-ray emission from IC 443 is detected at $\sim 86 \sigma$ level: the emission is extended with $68 \%$ containment angular radius $\theta_{68}^{\text {ext }}=0.27 \pm 0.01 \pm 0.03$ in the energy range between $1 \mathrm{GeV}$ and $50 \mathrm{GeV}$. The extension remains unchanged within error in the low $(1 \mathrm{GeV}<E<5 \mathrm{GeV})$ and high $(5 \mathrm{GeV}<E<50 \mathrm{GeV})$ energy bands.

2. The centroid of the emission moves at $\sim 1 \sigma-1.5 \sigma$ level toward that of the VERITAS source as the energy band changes from $1-5 \mathrm{GeV}$ to $5-50 \mathrm{GeV}$. The centroid is inconsistent with the PWN location, suggesting that the $\mathrm{PWN}$ is not the major contributor in the present energy range.

3. The centroid of the emission is consistent with that of EGRET (3EG J0617+2238), displaced more than $5 \times \theta_{68}^{\text {error }}(M A G I C)$ from that of MAGIC $(\mathrm{J} 0610+225)$, and at $1.5 \times \theta_{68}^{\text {error }}($ VERITAS) that of VERITAS (VER J0616.9+2230).

4. The extended source region overlaps almost completely that of VERITAS. A group of molecular clouds (Clouds B, C, $\mathrm{D}, \mathrm{F}$, and G), the SNR shell and the PWN are within the overlapping region $\left(\theta_{68}^{\text {ext }}\right)$, leaving possibility that some or all of them contribute to the observed emission.

5. The SED cannot be represented by a single power law but is consistent with a broken power law with a break at $E_{\gamma}=3.25 \pm 0.6 \mathrm{GeV}$.

6. The SED has a broad peak between a few $100 \mathrm{MeV}$ and $\sim 5 \mathrm{GeV}$ which is consistent with the majority of the emission coming from neutral pion decays. For the emission being hadronic originating from a single proton population, the underlying proton spectrum is consistent with a broken power-law shape (chi-square per degree of freedom of 9.6/14) but not with an exponential cutoff $(30.3 / 15)$. For the estimated total mass of interacting gas of $10^{4} M_{\odot}$, the total energy in the pion-producing protons is estimated to be $(0.56-2.2) \times 10^{49} \mathrm{erg}$.

Higher statistics is needed to establish association or nonassociation of the gamma-ray emission with the molecular clouds and/or the PWN as well as the CR injection process from the SNR into the Galactic space. Identification of the emission mechanisms and underlying CR spectra effective in individual sites will follow after such studies. 
Fermi LAT is expected to accumulate needed statistics well within the planned mission lifetime.

The Fermi LAT Collaboration acknowledges generous ongoing support from a number of agencies and institutes that have supported both the development and the operation of the LAT, as well as scientific data analysis. These include the National Aeronautics and Space Administration and the Department of Energy in the United States, the Commissariat à l'Energie Atomique and the Centre National de la Recherche Scientifique/Institut National de Physique Nucléaire et de Physique des Particules in France, the Agenzia Spaziale Italiana and the Istituto Nazionale di Fisica Nucleare in Italy, the Ministry of Education, Culture, Sports, Science and Technology (MEXT), High Energy Accelerator Research Organization (KEK), and Japan Aerospace Exploration Agency (JAXA) in Japan, and the K. A. Wallenberg Foundation, the Swedish Research Council, and the Swedish National Space Board in Sweden.

Additional support for science analysis during the operations phase is gratefully acknowledged from the Istituto Nazionale di Astrofisica in Italy and the Centre National d'Etudes Spatiales in France.

\section{REFERENCES}

Abdo, A. A., et al. 2009a, Astropart. Phys., 32, 193

Abdo, A. A., et al. 2009b, ApJS, 183, 46

Abdo, A. A., et al. 2009c, Phys. Rev. Lett., 103, 251101

Abdo, A. A., et al. 2009d, ApJ, 703, 1249

Acciari, V. A., et al. 2009, ApJ, 698, L133

Albert, J., et al. 2007, ApJ, 664, L87

Asaoka, I., \& Aschenbach, B. 1994, A\&A, 284, 573

Atwood, W., et al. 2009, ApJ, 697, 1071

Baring, M. G., Ellison, D. C., Reynolds, S. P., Grenier, I. A., \& Goret, P. 1999, ApJ, 513, 311

Beichman, C., et al. (eds.) 1988, IRAS Catalogs and Atlases, Vol. 1, Explanatory Supplement (RP-1190; Washington, DC: GPO)

Bocchino, F., \& Bykov, A. M. 2000, A\&A, 362, L29

Bocchino, F., \& Bykov, A. M. 2001, A\&A, 376, 248

Braun, R., \& Strom, R. G. 1986a, A\&AS, 63, 345

Braun, R., \& Strom, R. J. 1986b, A\&A, 164, 193

Burton, M. G., Geballe, T. R., Brand, P. W. J. L., \& Webster, A. S. 1988 MNRAS, 231, 617

Bykov, A. M., Chevalier, R. A., Ellison, D. C., \& Uvarov, Y. A. 2000, ApJ, 538, 203

Bykov, A. M., et al. 2008, ApJ, 676, 1050

Chevalier, R. A. 1999, ApJ, 511, 798

Cornett, R. H., Chin, G., \& Knapp, G. R. 1977, A\&A, 54, 889
Dame, T., Hartmann, D., \& Thaddeus, P. 2001, ApJ, 547, 792

Denoyer, L. K. 1978, MNRAS, 183, 187

Denoyer, L. K. 1979a, ApJ, 228, L41

Denoyer, L. K. 1979b, ApJ, 232, L165

Denoyer, L. K., \& Frerking, M. A. 1981, ApJ, 246, L37

Dickman, R. L., Snell, R. L., Ziurys, L. M., \& Huang, Y.-L. 1992, ApJ, 400, 203

Dobashi, K., et al. 2005, PASJ, 57, S1

Esposito, J. A., Hunter, S. D., Kanbach, G., \& Sreekumar, P. 1996, ApJ, 461, 820

Fesen, R. A., \& Kirshner, R. P. 1980, ApJ, 242, 1023

Frail, D. A., Goss, W. M., Reynoso, E. M., Giacani, E. B., Green, A. J., \& Otrupcek, R. 1996, AJ, 111, 1651

Furst, E., Reich, W., Reich, P., \& Reif, K. 1990, A\&AS, 85, 691

Gaensler, B. M., et al. 2006, ApJ, 648, 1037

Gaisser, T. K., \& Schaefer, R. K. 1992, ApJ, 394, 174

Giovanelli, R., \& Haynes, M. P. 1979, ApJ, 230, 404

Green, D. A. 2004, Bull. Astron. Soc. India, 32, 335G

Hartman, R. C., et al. 1999, ApJS, 123, 79

Hewitt, J. W., et al. 2006, ApJ, 652, 1288

Hewitt, J. W., et al. 2008, ApJ, 683, 189

Huang, Y.-L., Dickman, R. L., \& Snell, R. L. 1986, ApJ, 302, L63

Kalberla, P. M. W., et al. 2005, A\&A, 440, 775

Kamae, T., et al. 2006, ApJ, 647, 692 (erratum 662, 779 [2007])

Keohane, J. W., Petre, R., Gotthelf, E. V., Ozaki, M., \& Koyama, K. 1997, ApJ, 484,350

Lamb, R. C., \& Macomb, D. J. 1997, ApJ, 488, 872

Leahy, D. A. 2004, AJ, 127, 2277

Lee, J.-J., et al. 2008, AJ, 135, 796

Lozinskaya, T. A. 1981, Sov. Astron. Lett., 7, 17

Olbert, C. M., Clearfield, C. R., Williams, N. E., Keohane, J. W., \& Frail, D. A. 2001, ApJ, 554, L205

Petre, R., Szymkowiak, A. E., Seward, F. D., \& Willingale, R. 1988, ApJ, 335, 215

Ptuskin, V. S., \& Zirakashvili, V. N. 2005, A\&A, 429, 755

Richter, M. J., Graham, J. R., \& Wright, G. S. 1995, ApJ, 454, 277

Seta, M., et al. 1998, ApJ, 505, 286

Snell, R. L., et al. 2005, ApJ, 620, 758

Strong, A. W., \& Moskalenko, I. V. 1998, ApJ, 509, 212

Strong, A. W., Moskalenko, I. V., \& Reimer, O. 2001, ApJ, 537, 763

Strong, A. W., et al. 2009, arXiv:0907.0559

Sturner, S. J., \& Dermer, C. D. 1995, A\&A, 293, L17

Sturner, S. J., Skibo, J. G., Dermer, C. D., \& Mattox, J. R. 1997, ApJ, 490, 619

Torres, D. F., Rodriguez Marrero, A. Y., \& de Cea del Pozo, E. 2008, MNRAS 387, L59

Torres, D. F., Romero, G. E., Dame, T. M., Combi, J. A., \& Butt, Y. M. 2003, Phys. Rep., 382, 303

Troja, E., Bocchino, F., Miceli, M., \& Reale, F. 2008, A\&A, 485, 777

Troja, E., Bocchino, F., \& Reale, F. 2006, ApJ, 649, 258

van Dishoeck, E. F., Jansen, D. J., \& Phillips, T. G. 1993, A\&A, 279, 541

Welsh, B. Y., \& Sallmen, S. 2003, A\&A, 408, 545

Woltjer, L. 1972, ARA\&A, 10, 129

Zhang, L., \& Fang, J. 2008, ApJ, 675, L21 